Departamento de Zoologia Médica e Parasitologia

Prof. Dr. Z. Vaz

\title{
PARALLINTOSHIUS PARALLINTOSHIUS n. g., n. sp., (NEMATODA TRICHOSTRONGYLIDAE) PARASITA DE CHIROPTERA
}

\author{
POR \\ T. L. de Araujo \\ Assistente \\ Com 11 figuras
}

As necropsias que realizámos em morcegos de Casa Grande, S. Paulo, nos deram ocasião de encontrar uma espécie de nematoide que pensamos pertencer a um novo gênero. Obtivemos material relativamente abundante e em boas condições de estudo. Propomos para essa espécie a denominação de Parallintoshius parallintoshius $n$. g., n. sp., devido ao seu parentesco com as espécies do gênero Allintoshius Chitwood, 1937, descritas de morcegos dos Estados Unidos.

Consignamos aqui nossos agradecimentos ao Dr. Paulo C. Freire pelas facilidades que nos tem proporcionado na obtenção de material de Casa Grande e ao Snr. Carlos Vieira do Museu Paulista pela determinação dos hospedadores.

\section{Parallintostoshius n. g.}

Strongylacanthinae? Cutícula com estriação transversal fina, sem linhas longitudinais. Extremidade cefálica com dilatação cuticular. $\mathrm{Pa}$ pilas cervicais ausentes. Azas cervicais pouco desenvolvidas. Esôfago claviforme alongado.

Fêmeas com vulva provida de dois lábios muito salientes, situada na porção posterior do corpo. Vagina curta. Ovejetor bem desenvolvido, com ramos iguais; úteros opostos. Cauda estreitando-se abruptamente, terminando em ponta fina e com tres delicadas projeções cuticulares com aspecto de papilas, sub-ventrais.

Macho com bolsa copuladora ampla, trilobada, sem espinhos cuticulares. Lóbo dorsal muito pouco pronunciado. Fórmula bursal: ráios ventrais divergentes, ventro-ventral menor que o ventro-lateral; ráios laterais divergentes, saindo de tronco comum; lateral anterior no terço distal voltado para diante; laterais médio e posterior no terço distal voltados para trás; dorsal externo longo e muito fino nascendo em tronco comum com o dorsal; dorsal terminando por pequenas digitações, Espículos sub-iguais, curtos e grossos com pequenas azas. Gubernáculo presente. 
Habitat - Intestino delgado de Chiroptera.

Espécie tipo - Parallintoshius parallintoshius.

Parallintoshius parallintoshius n. sp.

Comprimento: macho 2,5-2,9 mm. fêmea $5,0-7,9 \mathrm{~mm}$.

Largura: 》 $0,086 \mathrm{~mm}$. 》 $0,11-0,14 \mathrm{~mm}$.

Nematóides levemente avermelhados quando vivos. Cutícula apresentando fina estriação transversal, sem linhas longitudinais. Extremidade anterior com dilatação cuticular assimétrica, mais desenvolvida no lado dorsal. Essa dilatação atinge um comprimento de $0,082-0,99 \mathrm{~mm}$. por $0,020-0,045 \mathrm{~mm}$. de largura máxima na fêmea e de 0,086 por $0,041 \mathrm{~mm}$. nos machos. Boca circular não seguida de cápsula bucal. Esôfago claviforme, alongado, medindo $0,24-0,29 \mathrm{~mm}$. de comprimento no macho e $0,35-0,37 \mathrm{~mm}$. na fêmea. A largura máxima do esôfago, no terço posterior, é de $0,020-0,024 \mathrm{~mm}$. no macho e de $0,020-0,028 \mathrm{~mm}$. na fêmea. Anel nervoso situado a $0,186-0,207 \mathrm{~mm}$. da extremidade anterior. Poro excretor de dificil observação colocado ao nivel do terço posterior do esôfago.

Fêmea com a vulva abrindo-se de $1,027 \mathrm{~mm}$. a $1,051 \mathrm{~mm}$. da extremidade posterior do corpo e circundada por dois lábios muito desenvolvidos. Ovejetor com vagina curta dirigida transversalmente. Ramos do ovejetor opostos e de comprimento igual, medem dum esfincter ao outro $0,09 \mathrm{~mm}$. Ovos larvados com $0,041-0,062 \mathrm{~mm}$. por $0,029 \mathrm{~mm}$. e larvas nos úteros. Corpo terminando por um apêndice delicado de $0,008 \mathrm{~mm}$. de comprimento, de posição ventral e por tres pequenas projeções cuticulares. Anus distando $0,07-0,14 \mathrm{~mm}$. da extremidade posterior.

Machos com bolsa copuladora de lóbos laterais bem desenvolvidos e com o dorsal muito pequeno. Ráios ventrais nascendo de tronco comum, divergindo em seguida. Ráio ventro-ventral dirigido para diante, não atingindo a margem bursal. Ráio ventro-lateral atingindo a margem bursal. Ráios laterais nascendo de tronco comum, contíguos e paralelos até à metade do comprimento, divergindo em seguida. Ráio lateral anterior dirigindo-se na porção terminal, para diante e não atingindo o bordo da bolsa. Lateral médio dirigido, no terço final para trás e atingindo a margem da bolsa. Lateral posterior dirigido, no terço terminal para trás, terminando na margem bursal junto ao dorsal externo. Dorsal externo muito longo e delgado, nascendo de tronco comum com o dorsal e terminando junto ao dorsal externo. Este ráio é muito delgado principalmente na metade proximal, alarga-se um pouco na metade distal. Ráio dorsal com $0,057 \mathrm{~mm}$. 
de comprimento, grosso na base, apresentando ramos laterais sub-terminais, bifurcando-se na extremidade, cada ramo da bifurcação terminando por duas pequenas digitações. Espículos simples, curtos, subiguais, medindo respectivamente $0,11-0,12 \mathrm{~mm}$. e $0,12-0,14 \mathrm{~mm}$. de comprimento. A forma geral dos espículos, quando vistos de frente, lembra um triângulo alongado e sua largura máxima, na extremidade anterior, varia entre $0,012-0,06 \mathrm{~mm}$. A extremidade posterior é afilada. Gubernáculo alongado com 0,053 mm. por 0,006 mm.

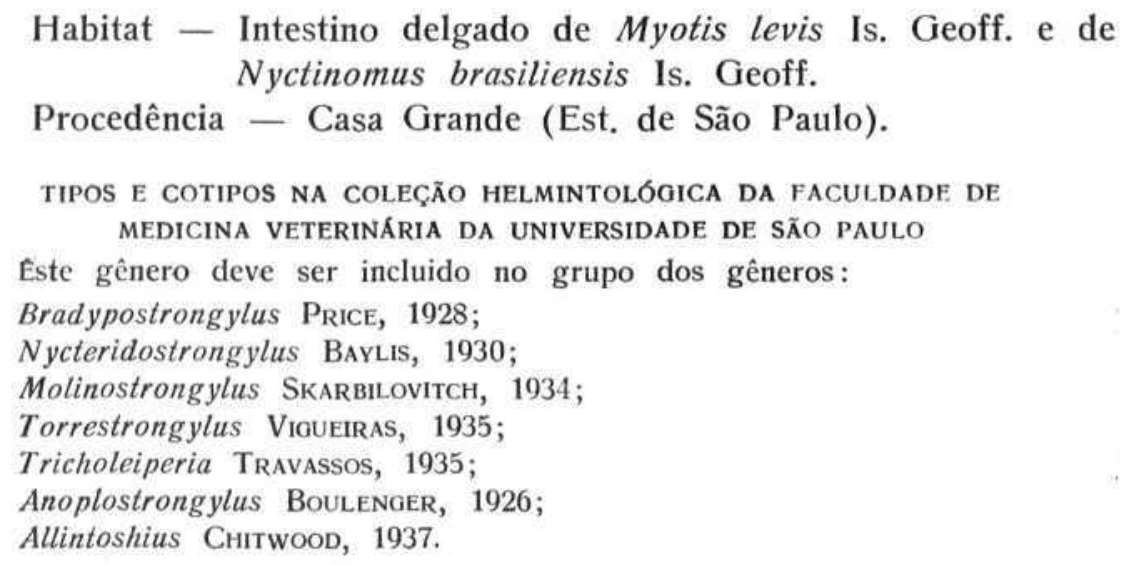
Nyctinomus brasiliensis Is. Geoff.

Procedência - Casa Grande (Est. de São Paulo).

TIPOS E COTIPOS NA COLEÇÃo HELMINTOLÓGICA DA FACULDADE DE MEDICINA VETERINÁRIA DA UNIVERSIDADE DE SÃO PAULO

Este gênero deve ser incluido no grupo dos gêneros:

Bradypostrongylus PRICE, 1928;

Nycteridostrongylus BAYLIS, 1930;

Molinostrongylus SKaRBILOVITCH, 1934;

Torrestrongylus VigueIRAS, 1935;

Tricholeiperia Travassos, 1935;

Anoplostrongylus BOULENGER, 1926;

Allintoshius CHIrwood, 1937.

$\mathrm{O}$ aspecto do ráio dorsal externo, extremamente delgado e longo e a presença de lábios vulvares muito salientes afasta-o dos gêneros acima. A forma dos espículos aproxima-o de Allintoshius CHItwood, 1937, do qual todavia bem se distingue pelos caracteres acima assinalados e ainda pelo aspecto da cauda da fêmea.

Deve-se acrescentar que o gênero ora proposto não possue verdadeiros espinhos na cauda da fêmea. Vêm-se delicadas espansões cuticulares com aspecto de papilas. Parece portanto uma forma de transição entre Allintoshius CHITwood. 1937, totalmente desprovido de espinhos na cauda da fêmea e os demais gêneros do grupo que todos êles mostram essas formações.

\section{ABSTRACT}

Parallintoshius parallintoshius n. g., n. sp., of Trichostrong y lidae, is described from brasilian bats (Myotis levis Is. Geoff. and Myctinomus brasiliensis Is. Geoff.) with the following generic diagnosis: Strongylacanthinae? Cuticle with fine transverse striations, without longitudinal ridges. Cephalic extremity with dilated cuticle. No cervical papillae seen. Lateral alae narrow. Oesophagus elongated, club-shaped.

Female: vulva posterior, with two proeminent lips. Vagina short. Ovejectors well developed. Uteri divergent. Tail narrowing abruptly, ending in a point, with three small e cuticular papillae-shaped projections. 
Male: with large, trilobed bursa without cuticular spines. Bursa with two large lateral lobes and a small dorsal one, with the following formula: ventral rays divergent, arising from a common trunk; externo lateral ray curving anteriorly at distal third; medio and posterolateral rays curving ventrally at distal third; externo dorsal ray long and uncommonly slender, arising from a common trunk with the dorsal one. Dorsal ray ending in small prongs. Spicules short and broard, with delicate alae. Gubernaculum present.

This new genus belongs to the group of the following genera:

Bradypostrongylus PRICE, 1928; Nycteridostrongylus Baylis, 1930, Molinostrongylus SKarbilovitch, 1934; Torrestrong y lus Vigueiras, 1935; Trichole i peria Travassos, 1935; Anoplostrongylus Boulenger, 1926 and Allintoshius CHitwood, 1937.

The very thin and long externo-dorsal ray and the presence of proeminent vulvar lips separate it from all the other genera in the group. The shape of the spicules relates it to Allintoshius Chitwood, 1937, from which it is easely distinguished by the above characters and by the shape of the female tail.

The females of this genus have no true spines in the tail, but only fine cuticular papillae-shaped projections. It seems to the A. that this new genus is a transition form between Allintoshius CHITwood, 1937, without spines in the female tail and the other genera of the group with such structures.

\section{BIBLIOGRAFIA}

Travassos L. - 1937 - Revisão da família Trichostrongylidae Leiper, 1912. - Monog. Inst. Osvaldo Cruz 1.

Travassos L. - 1921 - Contribuições para o conhecimento da fauna helmintológica brasileira. Ensaio Monografico da Família Triochostrongylidae. - Mem. Inst. Osw. Cruz XIII (1).

Chandler C. Asa - 1937 - A Report on the Parasites of a bat Mycticeius humeralis, with descriptions of four new helminths in Livro Jubilar do prof. L. Travassos.

YORK and MAPLESTONE - 1926 - The Nematode parasites of vertebrates. - Londres.

\section{EXPLICAÇÃO DAS FIGURAS}

Figs, 1 e 2 - Bolsa copuladora.

Fig. 3 - Espículos e gubernáculo.

Figs. 4, 5 e 6 - Variações do ráio dorsal.

Fig. 7 - Extremidade anterior, vista de lado.

" 8 - Região vulvar, vista de lado.

" 9 - Extremidade posterior da fêmea, vista de lado.

" 10 - Extremidade posterior da fêmea, vista de frente.

" 11 - Vulva e lábios de perfil. 
T. L. de Araujo - Parallintoshius parallintoshius n. g., n. sp.,

209
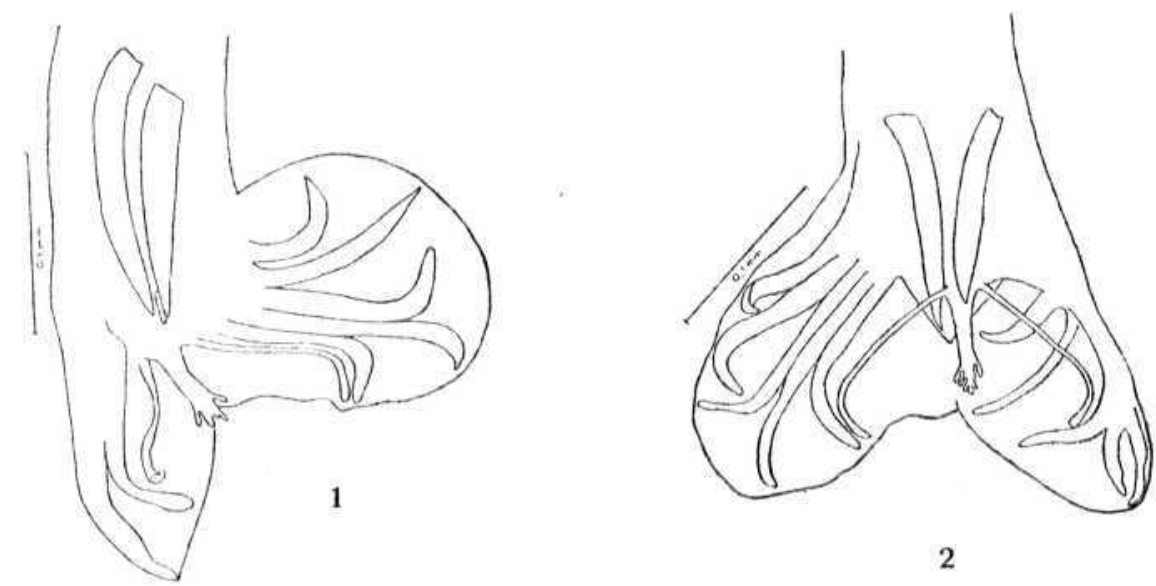

2

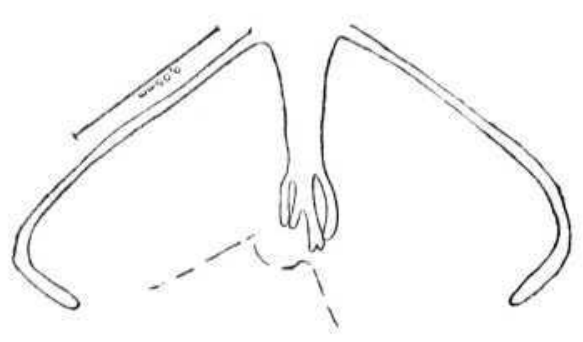

4

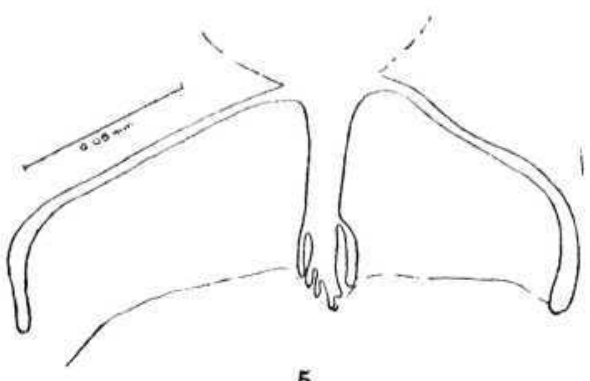

5

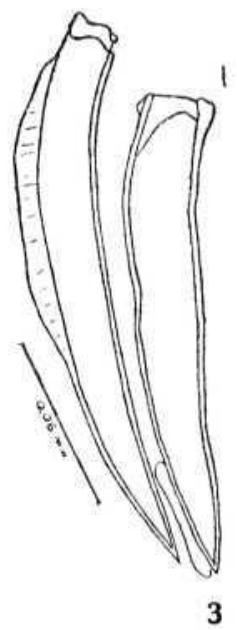




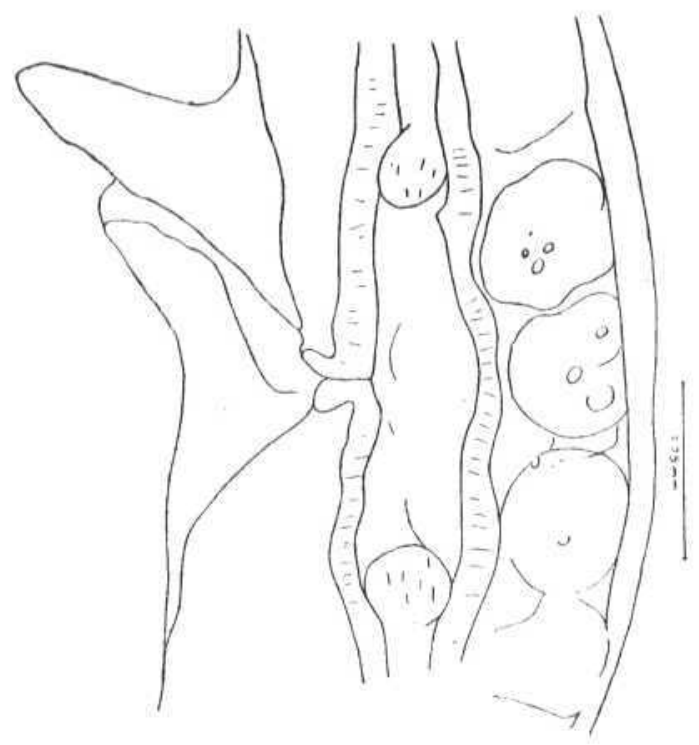

11
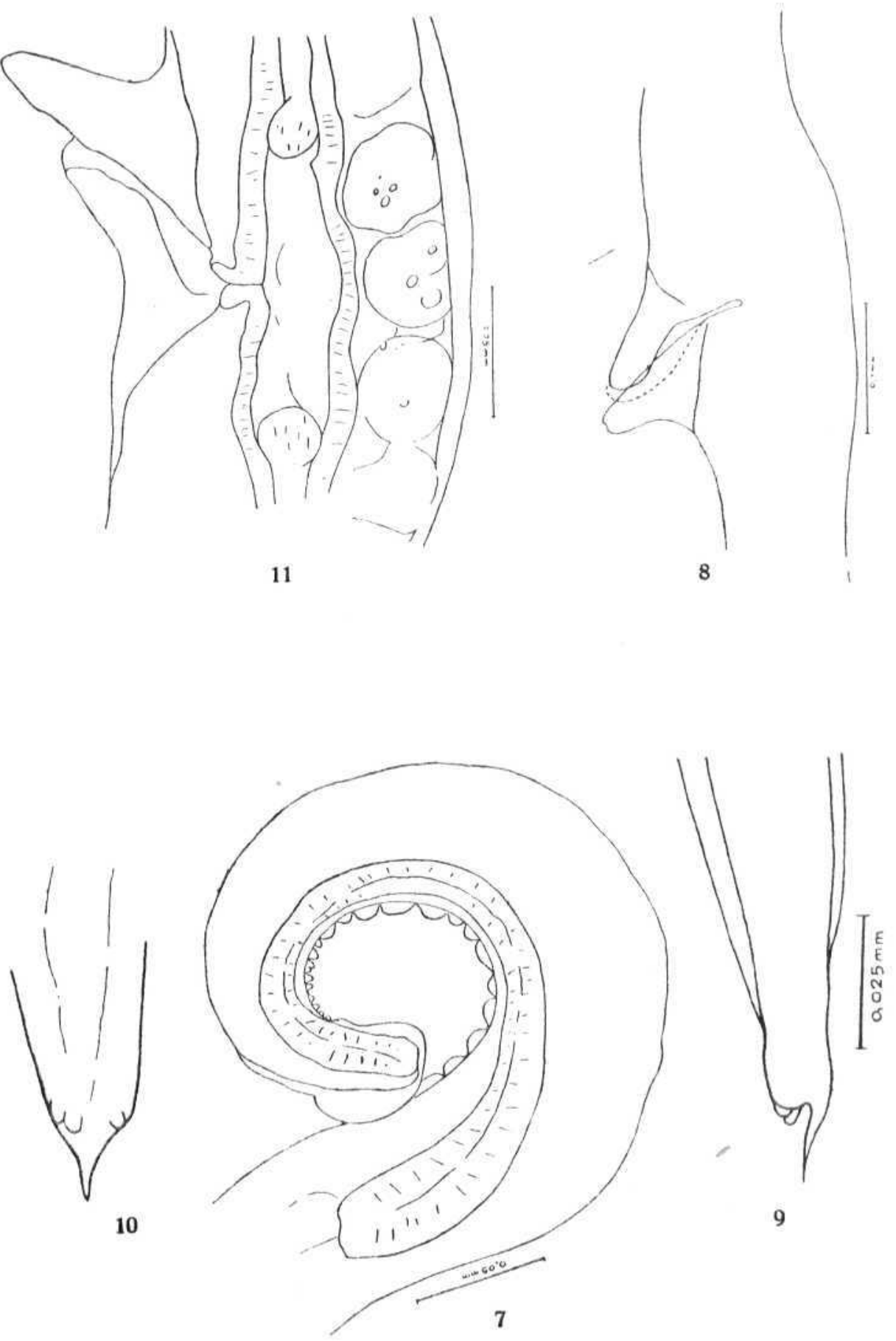